Theoretical and Mathematical Physics, 166(1): 81-93 (2011)

\title{
ESSENTIAL SPECTRUM OF A MODEL OPERATOR ASSOCIATED WITH A THREE-PARTICLE SYSTEM ON A LATTICE
}

\author{
(c) T. Kh. Rasulov*
}

\begin{abstract}
We consider a model operator $H$ associated with the system of three particles interacting via nonlocal pair potentials on a $\nu$-dimensional lattice. We identify channel operators and use their spectra to describe the position and structure of the essential spectrum of $H$. We obtain an analogue of the Faddeev equation for the eigenfunctions of $H$.
\end{abstract}

Keywords: model operator, nonlocal potential, Hilbert-Schmidt class, Faddeev equation, essential spectrum, channel operator

\section{Introduction}

Numerous works are devoted to investigating the essential spectrum of continuous and discrete Schrödinger operators (respectively see, e.g., [1]-[3] and [4]-[7]). In [5], in particular, it was proved that the essential spectrum of a three-particle discrete Schrödinger operator is the union of at most finitely many intervals even in the case where the corresponding two-particle discrete Schrödinger operator has an infinite number of eigenvalues.

In the physical literature, "local" potentials, i.e., operators of multiplication by a function, are typically used. But the potentials constructed, for example, in pseudopotential theory [8] turn out to be nonlocal and (also for a periodic operator) are given by the sum of a local and a finite-dimensional potential.

Here, we consider a model operator $H$ associated with a system of three particles on a $\nu$-dimensional lattice that interact via nonlocal pair potentials with the role of a two-particle discrete Schrödinger operator played by the Friedrichs model (see, e.g., [6]). We note that the operator $H$ can be regarded as a noncompact perturbation of the operator investigated in [6], [7], [9]. We identify channel operators. We describe the position and the structure of the essential spectrum of $H$ in terms of the spectrum of the channel operators. Moreover, we prove that the essential spectrum of this operator is a union of at most five intervals. We obtain an analogue of the Faddeev equation for eigenfunctions of $H$. The appearance of two-particle branches on both sides of the three-particle branch of the essential spectrum of $H$ (see Theorem 2.3 below) plays an important role in studying the finiteness or infiniteness of the discrete spectrum parts located there and in the gaps of the essential spectrum.

We note that the two-particle and three-particle branches of the essential spectrum of the three-particle continuous Schrödinger operator [1]-[3] are semi-infinite lines, which have intersections. In our situation, in contrast to the continuous case, such branches of the essential spectrum of $H$ fill finite-length intervals and may not intersect, i.e., a gap appears. We must therefore study the branches of the essential spectrum on both sides of the three-particle branch, which underlies the existence of the two-sided Efimov effect. It was proved in [4]-[7], [9] that the considered lattice operators do not have parts of the essential and discrete

*Bukhara State University, Bukhara, Uzbekistan; Institute for Mathematics, University of Bern, Bern, Switzerland, e-mail: rth@mail.ru, tulkin.rasulov@math.unibe.ch.

Translated from Teoreticheskaya i Matematicheskaya Fizika, Vol. 166, No. 1, pp. 95-109, January, 2011. Original article submitted May 16, 2010; revised June 16, 2010. 
spectra to the right of the three-particle branch. In those papers, investigating the essential spectrum position was based on the monotonicity of the Fredholm determinant in the Friedrichs model. In our case, unlike in previous works, the Fredholm determinant is not monotonic, and the investigation method is based on the number of eigenvalues in the Friedrichs model (see Lemmas 3.2-3.4 below).

This paper is organized as follows. In Sec. 2, we consider the model operator as a bounded self-adjoint operator in a Hilbert space and state the main results. In Sec. 3, based on the decomposition into a direct operator integral, we reduce studying spectral properties of channel operators to studying spectral properties of the Friedrichs model. In Sec. 4, we obtain an analogue of the Faddeev equation for eigenfunctions of $H$. In Sec. 5, we prove our main results.

\section{Model operator and formulation of the main results}

Let $\mathbf{T}^{\nu} \equiv(-\pi ; \pi]^{\nu}$ be the $\nu$-dimensional cube with the opposite faces identified, and let $L_{2}\left(\mathbf{T}^{\nu}\right)$ and $L_{2}\left(\left(\mathbf{T}^{\nu}\right)^{2}\right)$ be the respective Hilbert spaces of square-integrable (complex-valued) functions defined on $\mathbf{T}^{\nu}$ and $\left(\mathbf{T}^{\nu}\right)^{2}$. We consider the model operator $H$ acting in the Hilbert space $L_{2}\left(\left(\mathbf{T}^{\nu}\right)^{2}\right)$ as

$$
H=H_{0}-V_{1}-V_{2}
$$

with the operators $H_{0}$ and $V_{\alpha}, \alpha=1,2$, given by

$$
\begin{aligned}
& \left(H_{0} f\right)(p, q)=w(p, q) f(p, q), \\
& \left(V_{1} f\right)(p, q)=\int v_{1}(q, s) f(p, s) d s, \quad\left(V_{2} f\right)(p, q)=\int v_{2}(p, s) f(s, q) d s .
\end{aligned}
$$

Here, the function $v_{\alpha}(\cdot, \cdot), \alpha=1,2$, has the form

$$
v_{\alpha}(p, q)=v_{\alpha 1}(p) v_{\alpha 1}(q)-v_{\alpha 2}(p) v_{\alpha 2}(q), \quad \alpha=1,2,
$$

and $v_{i j}(\cdot), i, j=1,2$, and $w(\cdot, \cdot)$ are respectively real-valued continuous functions on $\mathbf{T}^{\nu}$ and $\left(\mathbf{T}^{\nu}\right)^{2}$. Here and hereafter, an integral with no integration limits denotes integration over the entire domains of the variables. Under these assumptions, the operator $H$ is bounded and self-adjoint in the Hilbert space $L_{2}\left(\left(\mathbf{T}^{\nu}\right)^{2}\right)$.

We note that nonlocal potentials with a degenerate kernel of the form

$$
\mathcal{V}(p, q)=-\sum_{i=1}^{n} f_{i}(p) g_{i}(q)
$$

were studied in [10], [11], where they were regarded as models associated with a system of several particles interacting via nonlocal pair potentials. For example, one of the nonlocal potentials is given by the Gauss potential, whose kernel has the form

$$
\mathcal{V}(p, q)=-\mu e^{-\beta\left(p^{2}+q^{2}\right) / 2}, \quad \mu, \beta>0,
$$

in the one-particle case.

Because two-particle Schrödinger equations are easily solved for nonlocal interactions, they are often used in nuclear physics and in multiparticle problems. They are also systematically used along with Faddeev equations for three-particle systems. The main feature of these equations [11] is that the particle-wave $t$ matrix retains its simple form and can easily be continued, which is the most important characteristic in nuclear physics and in Faddeev equations. 
We set

$$
w_{1}(p, q)=w(p, q), \quad w_{2}(p, q)=w(q, p) .
$$

Unless stipulated otherwise, we assume that $\alpha$ takes the values 1 and 2 . We let $\sigma(\cdot), \sigma_{\text {ess }}(\cdot)$, and $\sigma_{\text {disc }}(\cdot)$ denote the respective spectrum, essential spectrum, and discrete spectrum of a bounded self-adjoint operator.

To formulate our main results, along with the operator $H$, we also consider a bounded self-adjoint operator $H_{\alpha}$ acting in the Hilbert space $L_{2}\left(\left(\mathbf{T}^{\nu}\right)^{2}\right)$ as

$$
H_{\alpha}=H_{\alpha}^{0}-V_{\alpha}, \quad\left(H_{\alpha}^{0} f\right)(p, q)=w_{\alpha}(p, q) f(p, q),
$$

and the operator (Friedrichs model) $h_{\alpha}(p), p \in \mathbf{T}^{\nu}$, acting in $L_{2}\left(\mathbf{T}^{\nu}\right)$ as

$$
h_{\alpha}(p)=h_{\alpha}^{0}(p)-v_{\alpha}
$$

with the operators $h_{\alpha}^{0}(p), p \in \mathbf{T}^{\nu}$, and $v_{\alpha}$ defined as

$$
\left(h_{\alpha}^{0}(p) f\right)(q)=w_{\alpha}(p, q) f(q), \quad\left(v_{\alpha} f\right)(q)=\int v_{\alpha}(q, s) f(s) d s .
$$

The perturbation operator $v_{\alpha}$ of $h_{\alpha}^{0}(p), p \in \mathbf{T}^{\nu}$, is a self-adjoint two-dimensional operator. It follows from the known Weyl theorem [1] on preservation of the essential spectrum under finite-rank perturbations that the essential spectrum $\sigma_{\text {ess }}\left(h_{\alpha}(p)\right)$ of $h_{\alpha}(p), p \in \mathbf{T}^{\nu}$, coincides with the essential spectrum of $h_{\alpha}^{0}(p)$, $p \in \mathbf{T}^{\nu}$. It is known that $\sigma_{\text {ess }}\left(h_{\alpha}^{0}(p)\right)=\left[m_{\alpha}(p) ; M_{\alpha}(p)\right]$, where the numbers $m_{\alpha}(p)$ and $M_{\alpha}(p)$ are defined as

$$
m_{\alpha}(p)=\min _{q \in \mathbf{T}^{\nu}} w_{\alpha}(p, q), \quad M_{\alpha}(p)=\max _{q \in \mathbf{T}^{\nu}} w_{\alpha}(p, q) .
$$

It follows from the last two facts that $\sigma_{\mathrm{ess}}\left(h_{\alpha}(p)\right)=\left[m_{\alpha}(p) ; M_{\alpha}(p)\right]$.

Let $\mathbf{C}$ be the complex plane. For each fixed $p \in \mathbf{T}^{\nu}$, we define a function, regular in $\mathbf{C} \backslash\left[m_{\alpha}(p) ; M_{\alpha}(p)\right]$, as

$$
\Delta_{\alpha}(p ; z)=\Delta_{\alpha}^{(1)}(p ; z) \Delta_{\alpha}^{(2)}(p ; z)+\left(\Delta_{\alpha}^{(3)}(p ; z)\right)^{2}
$$

(the Fredholm determinant associated with the operator $h_{\alpha}(p), p \in \mathbf{T}^{\nu}$ ), where

$$
\begin{aligned}
& \Delta_{\alpha}^{(1)}(p ; z)=1-\int \frac{v_{\alpha 1}^{2}(s) d s}{w_{\alpha}(p, s)-z}, \quad \Delta_{\alpha}^{(2)}(p ; z)=1+\int \frac{v_{\alpha 2}^{2}(s) d s}{w_{\alpha}(p, s)-z}, \\
& \Delta_{\alpha}^{(3)}(p ; z)=\int \frac{v_{\alpha 1}(s) v_{\alpha 2}(s) d s}{w_{\alpha}(p, s)-z} .
\end{aligned}
$$

It then follows that (see Lemma 3.1 below)

$$
\sigma_{\text {disc }}\left(h_{\alpha}(p)\right)=\left\{z \in \mathbf{C} \backslash\left[m_{\alpha}(p), M_{\alpha}(p)\right]: \Delta_{\alpha}(p ; z)=0\right\}, \quad p \in \mathbf{T}^{\nu} .
$$

We set

$$
\begin{aligned}
& m=\min _{p, q \in \mathbf{T}^{\nu}} w(p, q), \quad M=\max _{p, q \in \mathbf{T}^{\nu}} w(p, q), \\
& \sigma_{\mathrm{two}}\left(H_{\alpha}\right)=\bigcup_{p \in \mathbf{T}^{\nu}} \sigma_{\text {disc }}\left(h_{\alpha}(p)\right), \quad \sigma_{\text {three }}\left(H_{\alpha}\right)=[m ; M] .
\end{aligned}
$$

The spectrum of $H_{\alpha}$ is described in the following theorem. 
Theorem 2.1. The equality

$$
\sigma\left(H_{\alpha}\right)=\sigma_{\text {two }}\left(H_{\alpha}\right) \cup \sigma_{\text {three }}\left(H_{\alpha}\right)
$$

holds for the spectrum of the operator $H_{\alpha}$.

We now formulate the result that describes the position of the essential spectrum of $H$.

Theorem 2.2. The essential spectrum of the operator $H$ coincides with the union of the spectra of the operators $H_{1}$ and $H_{2}$, i.e., $\sigma_{\mathrm{ess}}(H)=\sigma\left(H_{1}\right) \cup \sigma\left(H_{2}\right)$. Moreover, the set $\sigma_{\mathrm{ess}}(H)$ is a union of at most five intervals.

It is obvious that for a given $H$, the operators $H_{1}$ and $H_{2}$ are uniquely selected by the property of decomposability into a direct operator integral (see Sec. 3 below).

According to Theorem 2.2, the operators $H_{1}$ and $H_{2}$ have the characteristic property of a channel operator of the corresponding Schrödinger operator (see, e.g., [4], [5]). We therefore call them the channel operators associated with $H$. We note that the channel operators $H_{1}$ and $H_{2}$ have a structure simpler than that of $H$, and Theorem 2.2 therefore plays an important role in further investigating the spectrum of $H$.

We set

$$
\sigma_{\text {two }}(H)=\sigma_{\text {two }}\left(H_{1}\right) \cup \sigma_{\text {two }}\left(H_{2}\right), \quad \sigma_{\text {three }}(H)=\sigma_{\text {three }}\left(H_{1}\right) .
$$

We now introduce new subsets of the essential spectrum of $H$.

Definition. The sets $\sigma_{\text {two }}(H)$ and $\sigma_{\text {three }}(H)$ are called the two-particle and the three-particle branches of the essential spectrum of $H$.

We next assume that $\nu=3$ and formulate the following condition for our further analysis. The necessary statement for the corresponding dimension is given without comments.

Main Condition. The function $w(\cdot, \cdot)$ has a respective nondegenerate minimum and maximum at the points $\left(p_{\min }^{(i)}, q_{\min }^{(i)}\right), i=\overline{1, n}, 1 \leq n<\infty$, and $\left(p_{\max }^{(j)}, q_{\max }^{(j)}\right), j=\overline{1, m}, 1 \leq m<\infty$, of a six-dimensional torus.

If the main condition is satisfied, then the continuity of the function $v_{\alpha i}(\cdot), i=1,2$, on $\mathbf{T}^{3}$ implies that there exist finite integrals

$$
\int \frac{v_{\alpha i}(s) v_{\alpha j}(s) d s}{w_{\alpha}(p, s)-m}, \quad \int \frac{v_{\alpha i}(s) v_{\alpha j}(s) d s}{w_{\alpha}(p, s)-M}, \quad i, j=1,2, \quad p \in \mathbf{T}^{3} .
$$

It follows from the theorem on the limit transition under the Lebesgue integral that for each $p \in \mathbf{T}^{3}$, there exist finite limits

$$
\lim _{z \rightarrow m-0} \Delta_{\alpha}(p ; z)=\Delta_{\alpha}(p ; m), \quad \lim _{z \rightarrow M+0} \Delta_{\alpha}(p ; z)=\Delta_{\alpha}(p ; M),
$$

and the functions $\Delta_{\alpha}(\cdot ; m)$ and $\Delta_{\alpha}(\cdot ; M)$ are therefore continuous on $\mathbf{T}^{3}$.

We set

$$
\begin{array}{ll}
a_{\alpha}=\min \left\{\sigma_{\mathrm{two}}\left(H_{\alpha}\right) \cap(-\infty ; m]\right\}, & b_{\alpha}=\max \left\{\sigma_{\mathrm{two}}\left(H_{\alpha}\right) \cap(-\infty ; m]\right\}, \\
c_{\alpha}=\min \left\{\sigma_{\mathrm{two}}\left(H_{\alpha}\right) \cap[M ;+\infty)\right\}, & d_{\alpha}=\max \left\{\sigma_{\mathrm{two}}\left(H_{\alpha}\right) \cap[M ;+\infty)\right\} .
\end{array}
$$

The following theorem describes the structure of the spectrum of $H_{\alpha}$. 
Theorem 2.3. Let the main condition be satisfied. We assume the following:

1. $\min _{p \in \mathbf{T}^{3}} \Delta_{\alpha}(p ; m) \geq 0$, and then
a. $\sigma\left(H_{\alpha}\right)=[m ; M]$ if $\min _{p \in \mathbf{T}^{3}} \Delta_{\alpha}(p ; M) \geq 0$, and
b. $\sigma\left(H_{\alpha}\right)=[m ; M] \cup\left[c_{\alpha} ; d_{\alpha}\right], c_{\alpha}>M$, if $\max _{p \in \mathbf{T}^{3}} \Delta_{\alpha}(p ; M)<0$;

2. $\max _{p \in \mathbf{T}^{3}} \Delta_{\alpha}(p ; m)<0$, and then
a. $\sigma\left(H_{\alpha}\right)=\left[a_{\alpha} ; b_{\alpha}\right] \cup[m ; M], b_{\alpha}<m$, if $\min _{p \in \mathbf{T}^{3}} \Delta_{\alpha}(p ; M) \geq 0$, and
b. $\sigma\left(H_{\alpha}\right)=\left[a_{\alpha} ; b_{\alpha}\right] \cup[m ; M] \cup\left[c_{\alpha} ; d_{\alpha}\right], b_{\alpha}<m$ and $c_{\alpha}>M$, if $\max _{p \in \mathbf{T}^{3}} \Delta_{\alpha}(p ; M)<0$.

We note that Theorems 2.2 and 2.3 taken together describe the position and the structure of the essential spectrum.

Remark 2.1. The class of functions $v_{i j}(\cdot), i, j=1,2$, and $w(\cdot, \cdot)$ satisfying the conditions in Theorem 2.3 is nonempty (see Lemma 3.5 below).

\section{Some spectral properties of the operators $H_{\alpha}$ and $h_{\alpha}(p)$, $p \in \mathrm{T}^{\nu}$}

In this section, we use a decomposition into the direct operator integral to reduce investigating spectral properties of the channel operator $H_{\alpha}$ to studying spectral properties of the Friedrichs model $h_{\alpha}(p), p \in \mathbf{T}^{\nu}$, defined in (2.1). We describe the discrete spectrum of the operator $h_{\alpha}(p), p \in \mathbf{T}^{\nu}$.

It can be easily verified that the operators $H_{1}$ and $H_{2}$ respectively commute with any operator of multiplication by a bounded function $u_{1}(p)$ and $u_{2}(q)$ in $L_{2}\left(\left(\mathbf{T}^{\nu}\right)^{2}\right)$. It follows from the decomposition of the space $L_{2}\left(\left(\mathbf{T}^{\nu}\right)^{2}\right)$ into a direct integral,

$$
L_{2}\left(\left(\mathbf{T}^{\nu}\right)^{2}\right)=\int \oplus L_{2}\left(\mathbf{T}^{\nu}\right) d p
$$

that $H_{\alpha}$ decomposes into the direct operator integral

$$
H_{\alpha}=\int \oplus h_{\alpha}(p) d p
$$

where the operator $h_{\alpha}(p), p \in \mathbf{T}^{\nu}$, is defined in (2.1). We note that identical fibers appear in the direct integral in decomposition (3.1). We establish a relation between eigenvalues of the operator $h_{\alpha}(p), p \in \mathbf{T}^{\nu}$, and zeros of the function $\Delta_{\alpha}(p ; \cdot)$. The following lemma holds.

Lemma 3.1. For each fixed $p \in \mathbf{T}^{\nu}$, a number $z \in \mathbf{C} \backslash \sigma_{\text {ess }}\left(h_{\alpha}(p)\right)$ is an eigenvalue of $h_{\alpha}(p)$ if and only if $\Delta_{\alpha}(p ; z)=0$.

Proof. Let $z \in \mathbf{C} \backslash \sigma_{\text {ess }}\left(h_{\alpha}(p)\right)$ be an eigenvalue of $h_{\alpha}(p), p \in \mathbf{T}^{\nu}$, and let $f \in L_{2}\left(\mathbf{T}^{\nu}\right)$ be the corresponding eigenfunction, i.e., the equation

$$
w_{\alpha}(p, q) f(q)-\int\left[v_{\alpha 1}(q) v_{\alpha 1}(s)-v_{\alpha 2}(q) v_{\alpha 2}(s)\right] f(s) d s=z f(q)
$$

has a nonzero solution $f \in L_{2}\left(\mathbf{T}^{\nu}\right)$. We note that for any $z \in \mathbf{C} \backslash \sigma_{\text {ess }}\left(h_{\alpha}(p)\right)$ and $q \in \mathbf{T}^{\nu}$, the relation $w_{\alpha}(p, q)-z \neq 0, p \in \mathbf{T}^{\nu}$, holds. From Eq. (3.2) for $f$, we then have

$$
f(q)=\frac{v_{\alpha 1}(q) C_{\alpha}^{(1)}-v_{\alpha 2}(q) C_{\alpha}^{(2)}}{w_{\alpha}(p, q)-z},
$$


where

$$
C_{\alpha}^{(i)}=\int v_{\alpha i}(s) f(s) d s, \quad i=1,2 .
$$

Substituting expression (3.3) in (3.4), we find that Eq. (3.2) has a nonzero solution if and only if the system of equations

$$
\begin{aligned}
& \left(1-\int \frac{v_{\alpha 1}^{2}(s) d s}{w_{\alpha}(p, s)-z}\right) C_{\alpha}^{(1)}+\int \frac{v_{\alpha 1}(s) v_{\alpha 2}(s) d s}{w_{\alpha}(p, s)-z} C_{\alpha}^{(2)}=0 \\
& -\int \frac{v_{\alpha 1}(s) v_{\alpha 2}(s) d s}{w_{\alpha}(p, s)-z} C_{\alpha}^{(1)}+\left(1+\int \frac{v_{\alpha 2}^{2}(s) d s}{w_{\alpha}(p, s)-z}\right) C_{\alpha}^{(2)}=0
\end{aligned}
$$

has a nonzero solution $\left(C_{\alpha}^{(1)}, C_{\alpha}^{(2)}\right) \in \mathbf{C}^{2}$, i.e., if $\Delta_{\alpha}(p ; z)=0$, where $\mathbf{C}^{2}$ is the Cartesian square of the set C. The lemma is proved.

For any bounded self-adjoint operator $A$ acting in a Hilbert space $\mathcal{H}$, we let $\mathcal{H}_{A}(z)$ denote the subspace such that $(A f, f)<\lambda\|f\|$ for any $f \in \mathcal{H}_{A}(z)$ and set

$$
N(\lambda, A)=\sup _{\mathcal{H}_{A}(z)} \operatorname{dim} \mathcal{H}_{A}(z)
$$

The number $N(\lambda, A)$ is equal to infinity if $\lambda>\min \sigma_{\text {ess }}(A)$; if $N(\lambda, A)$ is finite, then it is equal to the number of eigenvalues of $A$ that are less than $\lambda$, counted with multiplicities.

Lemma 3.2. For any $p \in \mathbf{T}^{\nu}$, the operator $h_{\alpha}(p)$ can have no more than one simple eigenvalue to the left of $m_{\alpha}(p)$ and to the right of $M_{\alpha}(p)$.

Proof. We introduce the operator $v_{\alpha i}, i=1,2$, acting in $L_{2}\left(\mathbf{T}^{\nu}\right)$ as

$$
\left(v_{\alpha i} f\right)(q)=v_{\alpha i}(q) \int v_{\alpha i}(s) f(s) d s, \quad i=1,2 .
$$

Then the operator $v_{\alpha}$ can be written as $v_{\alpha}=v_{\alpha 1}-v_{\alpha 2}$. We set $h_{\alpha}^{(1)}=h_{\alpha}^{0}-v_{\alpha 1}$. Because the operator $v_{\alpha i}$, $i=1,2$, is nonnegative, it is easy to show that $h_{\alpha}(p) \geq h_{\alpha}^{(1)}(p)$, and therefore

$$
\mathcal{H}_{h_{\alpha}(p)}(z) \subset \mathcal{H}_{h_{\alpha}^{(1)}(p)}(z), \quad z \leq m_{\alpha}(p)
$$

This implies that

$$
N\left(z, h_{\alpha}(p)\right) \leq N\left(z, h_{\alpha}^{(1)}(p)\right), \quad z \leq m_{\alpha}(p)
$$

Because the Fredholm determinant $\Delta_{\alpha}^{(1)}(p ; \cdot)$ of $h_{\alpha}^{(1)}(p)$ decreases monotonically on the half-axis $(-\infty$, $\left.m_{\alpha}(p)\right)$, it follows that $N\left(m_{\alpha}(p), h_{\alpha}^{(1)}(p)\right) \leq 1$. Therefore, by inequalities (3.5), the inequality

$$
N\left(m_{\alpha}(p), h_{\alpha}(p)\right) \leq 1
$$

holds. This implies that for any $p \in \mathbf{T}^{\nu}$, the operator $h_{\alpha}(p)$ can have no more than one simple eigenvalue to the left of $m_{\alpha}(p)$.

It can be shown similarly that $N\left(-M_{\alpha}(p),-h_{\alpha}(p)\right) \leq 1$. The lemma is proved.

The next lemma describes the set of eigenvalues of $h_{\alpha}(p), p \in \mathbf{T}^{3}$, on $(-\infty, m)$. 
Lemma 3.3. Let the main condition be satisfied. Then

1. if $\min _{p \in \mathbf{T}^{3}} \Delta_{\alpha}(p ; m) \geq 0$, then for any $p \in \mathbf{T}^{3}$, the operator $h_{\alpha}(p)$ has no eigenvalues on $(-\infty, m)$, and

2. if $\max _{p \in \mathbf{T}^{3}} \Delta_{\alpha}(p ; m)<0$, then for any $p \in \mathbf{T}^{3}$, the operator $h_{\alpha}(p)$ has a unique simple eigenvalue on $(-\infty, m)$.

Lemma 3.3 is proved similarly to the next lemma, which describes the set of eigenvalues of the operator $h_{\alpha}(p), p \in \mathbf{T}^{3}$, on $(M,+\infty)$.

Lemma 3.4. Let the main condition be satisfied. Then

1. if $\min _{p \in \mathbf{T}^{3}} \Delta_{\alpha}(p ; M) \geq 0$, then for any $p \in \mathbf{T}^{3}$, the operator $h_{\alpha}(p)$ has no eigenvalues on $(M,+\infty)$, and

2. if $\max _{p \in \mathbf{T}^{3}} \Delta_{\alpha}(p ; M)<0$, then for any $p \in \mathbf{T}^{3}$, the operator $h_{\alpha}(p)$ has a unique simple eigenvalue on $(M,+\infty)$.

Proof. 1. Let $\min _{p \in \mathbf{T}^{3}} \Delta_{\alpha}(p ; M) \geq 0$. Then the inequality $\Delta_{\alpha}(p ; M) \geq 0$ holds for any $p \in \mathbf{T}^{3}$. It follows from the continuity of the function $\Delta_{\alpha}(p ; \cdot)$ on $[M, \infty)$ and the equality

$$
\lim _{z \rightarrow+\infty} \Delta_{\alpha}(p ; z)=1
$$

that the function $\Delta_{\alpha}(p ; \cdot)$ either has no zeros on $(M, \infty)$ or has at least two zeros (counted with multiplicities) on $(M, \infty)$. In the second case, by Lemma 3.1 , the operator $h_{\alpha}(p)$ has at least two eigenvalues (counted with multiplicities) on $(M, \infty)$. This contradicts the statement of Lemma 3.2. Hence, for any $p \in \mathbf{T}^{3}$, the operator $h_{\alpha}(p)$ has no eigenvalues on $(M, \infty)$. We here also use the fact that a number $z_{0} \in \mathbf{C} \backslash \sigma_{\text {ess }}\left(h_{\alpha}(p)\right)$ is an eigenvalue of $h_{\alpha}(p)$ with multiplicity $n$ if and only if $z_{0}$ is a zero of the function $\Delta_{\alpha}(p ; \cdot)$ with multiplicity $n[12]$.

2. Let $\max _{p \in \mathbf{T}^{3}} \Delta_{\alpha}(p ; M)<0$. Then the inequality $\Delta_{\alpha}(p ; M)<0$ holds for any $p \in \mathbf{T}^{3}$. By (3.6), the function $\Delta_{\alpha}(p ; \cdot)$ for all $p \in \mathbf{T}^{3}$ has a unique simple zero on $(M, \infty)$ or has at least three zeros (counted with multiplicities) on $(M, \infty)$. Arguing similarly to the above, we conclude that only the first case is possible, which by Lemma 3.1 then implies that for all $p \in \mathbf{T}^{3}$, the operator $h_{\alpha}(p)$ has a unique simple eigenvalue on $(M,+\infty)$. Lemma 3.4 is proved.

Lemma 3.5. Let $w(\cdot, \cdot)$ be a function satisfying the main condition. We assume that $v_{\alpha i}(p)=$ $\sqrt{\mu_{\alpha i}} \hat{v}_{\alpha i}(p), \mu_{\alpha i}>0, i=1,2$, such that the function $\hat{v}_{\alpha i}(\cdot), i=1,2$, is continuous on $\mathbf{T}^{3}$ with a compact support $\Omega_{\alpha i} \subset \mathbf{T}^{3}$ and $\Omega_{\alpha 1} \cap \Omega_{\alpha 2}=\varnothing$. We set

$$
\begin{array}{ll}
\mu_{\alpha 1}^{(1)}=\min _{p \in \mathbf{T}^{3}}\left(\int_{\Omega_{\alpha 1}} \frac{\hat{v}_{\alpha 1}^{2}(s) d s}{w_{\alpha}(p, s)-m}\right)^{-1}, & \mu_{\alpha 1}^{(2)}=\max _{p \in \mathbf{T}^{3}}\left(\int_{\Omega_{\alpha 1}} \frac{\hat{v}_{\alpha 1}^{2}(s) d s}{w_{\alpha}(p, s)-m}\right)^{-1}, \\
\mu_{\alpha 2}^{(1)}=\min _{p \in \mathbf{T}^{3}}\left(\int_{\Omega_{\alpha 2}} \frac{\hat{v}_{\alpha 2}^{2}(s) d s}{M-w_{\alpha}(p, s)}\right)^{-1}, & \mu_{\alpha 2}^{(2)}=\max _{p \in \mathbf{T}^{3}}\left(\int_{\Omega_{\alpha 2}} \frac{\hat{v}_{\alpha 2}^{2}(s) d s}{M-w_{\alpha}(p, s)}\right)^{-1} .
\end{array}
$$

We consider the cases
a. $0<\mu_{\alpha 1} \leq \mu_{\alpha 1}^{(1)}$ and $0<\mu_{\alpha 2} \leq \mu_{\alpha 2}^{(1)}$,
b. $0<\mu_{\alpha 1} \leq \mu_{\alpha 1}^{(1)}$ and $\mu_{\alpha 2}>\mu_{\alpha 2}^{(2)}$, 
c. $\mu_{\alpha 1}>\mu_{\alpha 1}^{(2)}$ and $0<\mu_{\alpha 2} \leq \mu_{\alpha 2}^{(1)}$, and

d. $\mu_{\alpha 1}>\mu_{\alpha 1}^{(2)}$ and $\mu_{\alpha 2}>\mu_{\alpha 2}^{(2)}$.

If conditions $\mathrm{a}, \mathrm{b}, \mathrm{c}$, or $\mathrm{d}$ are satisfied, then the respective conditions $1 \mathrm{a}, 1 \mathrm{~b}, 2 \mathrm{a}$, and $2 \mathrm{~b}$ in the statement of Theorem 2.3 are also satisfied.

Remark 3.1. We note that examples of the functions $w(\cdot, \cdot)$ and $\hat{v}_{\alpha i}(\cdot), i=1,2$, in Lemma 3.5 are

$$
\begin{aligned}
& w(p, q)=\varepsilon(p)+4 \varepsilon\left(\frac{1}{2}(p+q)\right)+\varepsilon(q), \quad \varepsilon(p)=\sum_{i=1}^{3}\left(1-\cos p^{(i)}\right), \\
& p=\left(p^{(1)}, p^{(2)}, p^{(3)}\right) \in \mathbf{T}^{3}, \\
& \hat{v}_{\alpha 1}(p)= \begin{cases}\alpha \sum_{i=1}^{3}\left(\cos p^{(i)}-\cos 1\right), & p \in B_{1}(0), \\
0, & p \in \mathbf{T}^{3} \backslash B_{1}(0),\end{cases} \\
& \hat{v}_{\alpha 2}(p)= \begin{cases}\alpha \sum_{i=1}^{3}\left(\cos p^{(i)}+\cos 1\right), & p \in B_{1}(\bar{\pi}), \\
0, & p \in \mathbf{T}^{3} \backslash B_{1}(\bar{\pi}) .\end{cases}
\end{aligned}
$$

Here, $B_{1}\left(p_{0}\right)=\left\{p \in \mathbf{T}^{3}:\left|p-p_{0}\right| \leq 1\right\}, p_{0} \in \mathbf{T}^{3}$. It can be seen that the function $w(\cdot, \cdot)$ has a unique nondegenerate minimum at $(0,0) \in\left(\mathbf{T}^{3}\right)^{2}$ and a maximum at $(\bar{\pi}, \bar{\pi}) \in\left(\mathbf{T}^{3}\right)^{2}, \bar{\pi}=(\pi, \pi, \pi) \in \mathbf{T}^{3}$, i.e., $n=m=1$.

Proof of Lemma 3.5. We first note that with the conditions imposed on the functions $\hat{v}_{\alpha i}(\cdot)$, $i=1,2$, the equality $\Delta_{\alpha}(p ; z)=\Delta_{\alpha}^{(1)}(p ; z) \Delta_{\alpha}^{(2)}(p ; z)$ holds. We prove the lemma in case $c$. The other statements are proved similarly.

Let $\mu_{\alpha 1}>\mu_{\alpha 1}^{(2)}$ and $0<\mu_{\alpha 2} \leq \mu_{\alpha 2}^{(1)}$. Then

$$
\max _{p \in \mathbf{T}^{3}} \Delta_{\alpha}(p ; m) \leq \max _{p \in \mathbf{T}^{3}}\left(1-\mu_{\alpha 1} \int_{\Omega_{\alpha 1}} \frac{\hat{v}_{\alpha 1}^{2}(s) d s}{w_{\alpha}(p, s)-m}\right) \max _{p \in \mathbf{T}^{3}}\left(1+\mu_{\alpha 2} \int_{\Omega_{\alpha 2}} \frac{\hat{v}_{\alpha 2}^{2}(s) d s}{w_{\alpha}(p, s)-m}\right) .
$$

In the last inequality, the second factor in the right-hand side is positive for any $\mu_{\alpha 2}>0$. Because $\mu_{\alpha 1}>\mu_{\alpha 1}^{(2)}$, the first factor is negative, and hence $\max _{p \in \mathbf{T}^{3}} \Delta_{\alpha}(p ; m)<0$. Just the same argument shows that for any $\mu_{\alpha 1}>0$ and $0<\mu_{\alpha 2} \leq \mu_{\alpha 2}^{(1)}$, the inequality $\min _{p \in \mathbf{T}^{3}} \Delta_{\alpha}(p ; M) \geq 0$ holds. The lemma is proved.

\section{An analogue of the Faddeev equation for eigenfunctions of $\boldsymbol{H}$}

For eigenfunctions of the operator $H$, we here derive an analogue of the system of the Faddeev integral equations, which plays an important role in investigating the spectrum of this operator. Our constructions and argument are similar in part to those in [13], and whenever such a similarity occurs, we limit ourself to the corresponding reference.

Let $L_{2}^{(2)}\left(\mathbf{T}^{\nu}\right)$ be the Hilbert space of two-component vector functions $f=\left(f_{1}, f_{2}\right), f_{\alpha} \in L_{2}\left(\mathbf{T}^{\nu}\right)$, $\alpha=1,2$. For each $z \in \mathbf{C} \backslash \sigma_{\text {three }}(H)$, we introduce block operator matrices $A(z)$ and $K(z)$ acting in the space $L_{2}^{(4)}\left(\mathbf{T}^{\nu}\right)=L_{2}^{(2)}\left(\mathbf{T}^{\nu}\right) \oplus L_{2}^{(2)}\left(\mathbf{T}^{\nu}\right)$ as

$$
A(z)=\left(\begin{array}{cc}
A_{1}(z) & 0 \\
0 & A_{2}(z)
\end{array}\right), \quad K(z)=\left(\begin{array}{cc}
0 & K_{2}(z) \\
K_{1}(z) & 0
\end{array}\right)
$$


where the matrix elements $A_{\alpha}(z)$ and $K_{\alpha}(z)$ are also block operator matrices acting in the space $L_{2}^{(2)}\left(\mathbf{T}^{\nu}\right)$,

$$
A_{\alpha}(z)=\left(\begin{array}{cc}
A_{11}^{(\alpha)}(z) & A_{12}^{(\alpha)}(z) \\
A_{21}^{(\alpha)}(z) & A_{22}^{(\alpha)}(z)
\end{array}\right), \quad K_{\alpha}(z)=\left(\begin{array}{cc}
K_{11}^{(\alpha)}(z) & K_{12}^{(\alpha)}(z) \\
K_{21}^{(\alpha)}(z) & K_{22}^{(\alpha)}(z)
\end{array}\right) .
$$

Here, $A_{i j}^{(\alpha)}(z): L_{2}\left(\mathbf{T}^{\nu}\right) \rightarrow L_{2}\left(\mathbf{T}^{\nu}\right), i, j=1,2, z \in \mathbf{C} \backslash \sigma_{\text {three }}(H)$, is the operator of multiplication by the function $a_{i j}^{(\alpha)}(p ; z)$,

$$
\begin{aligned}
& a_{11}^{(\alpha)}(p ; z)=\Delta_{\alpha}^{(1)}(p ; z), \quad a_{12}^{(\alpha)}(p ; z)=-a_{21}^{(\alpha)}(p ; z)=\Delta_{\alpha}^{(3)}(p ; z), \\
& a_{22}^{(\alpha)}(p ; z)=\Delta_{\alpha}^{(2)}(p ; z),
\end{aligned}
$$

and $K_{i j}^{(\alpha)}(z): L_{2}\left(\mathbf{T}^{\nu}\right) \rightarrow L_{2}\left(\mathbf{T}^{\nu}\right), i, j=1,2, z \in \mathbf{C} \backslash \sigma_{\text {three }}(H)$, is the integral operator with the kernel $K_{i j}^{(\alpha)}(p, s ; z)$ ( $s$ is the integration variable),

$$
\begin{aligned}
& K_{11}^{(1)}(p, s ; z)=K_{11}^{(2)}(s, p ; z)=\frac{v_{11}(p) v_{21}(s)}{w_{2}(p, s)-z} \\
& K_{12}^{(1)}(p, s ; z)=-K_{21}^{(2)}(s, p ; z)=-\frac{v_{12}(s) v_{21}(q)}{w_{2}(p, s)-z} \\
& K_{21}^{(1)}(p, s ; z)=-K_{12}^{(1)}(s, p ; z)=\frac{v_{11}(s) v_{22}(q)}{w_{2}(p, s)-z} \\
& K_{22}^{(1)}(p, s ; z)=K_{22}^{(2)}(s, p ; z)=-\frac{v_{12}(p) v_{22}(s)}{w_{2}(p, s)-z}
\end{aligned}
$$

We note that for each $z \in \mathbf{C} \backslash \sigma_{\text {three }}(H)$, the operator $K_{i j}^{(\alpha)}(z), i, j=1,2$, belongs to the Hilbert-Schmidt class, and $K(z)$ is therefore a compact operator.

Lemma 4.1. For each $z \in \mathbf{C} \backslash\left(\sigma_{\text {two }}(H) \cup \sigma_{\text {three }}(H)\right)$, the operator $A(z)$ is bounded and invertible. The inverse operator $A^{-1}(z)$ is given by

$$
A^{-1}(z)=\left(\begin{array}{cc}
A_{1}^{-1}(z) & 0 \\
0 & A_{2}^{-1}(z)
\end{array}\right), \quad \text { where } A_{\alpha}^{-1}(z)=\left(\begin{array}{cc}
B_{11}^{(\alpha)}(z) & B_{12}^{(\alpha)}(z) \\
B_{21}^{(\alpha)}(z) & B_{22}^{(\alpha)}(z)
\end{array}\right) .
$$

Here, $B_{i j}^{(\alpha)}(z): L_{2}\left(\mathbf{T}^{\nu}\right) \rightarrow L_{2}\left(\mathbf{T}^{\nu}\right), i, j=1,2, z \in \mathbf{C} \backslash\left(\sigma_{\text {two }}(H) \cup \sigma_{\text {three }}(H)\right)$, is the operator of multiplication by the function $b_{i j}^{(\alpha)}(p ; z)$,

$$
\begin{array}{ll}
b_{11}^{(\alpha)}(p ; z)=\frac{a_{22}^{(\alpha)}(p ; z)}{\Delta_{\alpha}(p ; z)}, & b_{12}^{(\alpha)}(p ; z)=-\frac{a_{12}^{(\alpha)}(p ; z)}{\Delta_{\alpha}(p ; z)}, \\
b_{21}^{(\alpha)}(p ; z)=-\frac{a_{21}^{(\alpha)}(p ; z)}{\Delta_{\alpha}(p ; z)}, & b_{22}^{(\alpha)}(p ; z)=\frac{a_{11}^{(\alpha)}(p ; z)}{\Delta_{\alpha}(p ; z)} .
\end{array}
$$

Lemma 4.1 is proved similarly to the corresponding lemma in [13].

Because the operator $A(z)$ is invertible for each $z \in \mathbf{C} \backslash\left(\sigma_{\text {two }}(H) \cup \sigma_{\text {three }}(H)\right)$, the operator

$$
T(z)=A^{-1}(z) K(z)=\left(\begin{array}{cc}
0 & A_{1}^{-1}(z) K_{2}(z) \\
A_{2}^{-1}(z) K_{1}(z) & 0
\end{array}\right)
$$

is defined for such $z$. The following lemma establishes a relation between eigenvalues of the operators $H$ and $T(z)$. 
Lemma 4.2. A number $z \in \mathbf{C} \backslash\left(\sigma_{\text {two }}(H) \cup \sigma_{\text {three }}(H)\right)$ is an eigenvalue of the operator $H$ if and only if the operator $T(z)$ has a unit eigenvalue and their multiplicities coincide.

Proof. Let $z \in \mathbf{C} \backslash\left(\sigma_{\text {two }}(H) \cup \sigma_{\text {three }}(H)\right)$ be an eigenvalue of $H$ and $f \in L_{2}\left(\left(\mathbf{T}^{\nu}\right)^{2}\right)$ be the corresponding eigenfunction. Then the equation $H f=z f$ or the equation

$$
\begin{aligned}
(w(p, q)-z) f(p, q)- & \int\left[v_{11}(q) v_{11}(s)-v_{12}(q) v_{12}(s)\right] f(p, s) d s- \\
& -\int\left[v_{21}(p) v_{21}(s)-v_{22}(p) v_{22}(s)\right] f(s, q) d s=0
\end{aligned}
$$

has a nontrivial solution $f \in L_{2}\left(\left(\mathbf{T}^{\nu}\right)^{2}\right)$. Because $z \notin \sigma_{\text {three }}(H)$, it follows that $f$ in Eq. (4.1) is given by

$$
f(p, q)=\frac{v_{11}(q) g_{1}^{(1)}(p)-v_{12}(q) g_{2}^{(1)}(p)+v_{21}(p) g_{1}^{(2)}(q)-v_{22}(p) g_{2}^{(2)}(q)}{w(p, q)-z}
$$

where

$$
g_{\alpha}^{(1)}(p)=\int v_{1 \alpha}(s) f(p, s) d s, \quad g_{\alpha}^{(2)}(p)=\int v_{2 \alpha}(s) f(s, p) d s .
$$

Substituting expression (4.2) in (4.3), we find that the system of equations

$$
\begin{aligned}
& \Delta_{1}^{(1)}(p ; z) g_{1}^{(1)}(p)+\Delta_{3}^{(1)}(p ; z) g_{2}^{(1)}(p)= \\
& =v_{21}(p) \int \frac{v_{11}(s) g_{1}^{(2)}(s)}{w_{1}(p, s)-z} d s-v_{22}(p) \int \frac{v_{11}(s) g_{2}^{(2)}(s)}{w_{1}(p, s)-z} d s \\
& -\Delta_{3}^{(1)}(p ; z) g_{1}^{(1)}(p)+\Delta_{2}^{(1)}(p ; z) g_{2}^{(1)}(p)= \\
& =v_{21}(p) \int \frac{v_{12}(s) g_{1}^{(2)}(s)}{w_{1}(p, s)-z} d s-v_{22}(p) \int \frac{v_{12}(s) g_{2}^{(2)}(s)}{w_{1}(p, s)-z} d s \\
& \Delta_{1}^{(2)}(p ; z) g_{1}^{(2)}(p)+\Delta_{3}^{(2)}(p ; z) g_{2}^{(2)}(p)= \\
& =v_{11}(p) \int \frac{v_{21}(s) g_{1}^{(1)}(s)}{w_{2}(p, s)-z} d s-v_{12}(p) \int \frac{v_{21}(s) g_{2}^{(1)}(s)}{w_{2}(p, s)-z} d s \\
& -\Delta_{3}^{(2)}(p ; z) g_{1}^{(2)}(p)+\Delta_{2}^{(2)}(p ; z) g_{2}^{(2)}(p)= \\
& =v_{11}(p) \int \frac{v_{22}(s) g_{1}^{(1)}(s)}{w_{2}(p, s)-z} d s-v_{12}(p) \int \frac{v_{22}(s) g_{2}^{(1)}(s)}{w_{2}(p, s)-z} d s
\end{aligned}
$$

or the equation

$$
A(z) g=K(z) g, \quad g=\left(g_{1}^{(1)}, g_{2}^{(1)}, g_{1}^{(2)}, g_{2}^{(2)}\right) \in L_{2}^{(4)}\left(\mathbf{T}^{\nu}\right),
$$

has a nontrivial solution if and only if Eq. (4.1) has a nontrivial solution and the linear subspaces generated by solutions of Eqs. (4.1) and (4.4) have the same dimension.

By Lemma 4.1, the operator $A(z)$ is invertible for each $z \in \mathbf{C} \backslash\left(\sigma_{\text {two }}(H) \cup \sigma_{\text {three }}(H)\right)$. Therefore, the equation $g=A^{-1}(z) K(z) g$, i.e., the equation $g=T(z) g$, has a nontrivial solution if and only if Eq. (4.4) has a nontrivial solution. Here, too, the linear subspaces generated by solutions of Eqs. (4.4) and $g=T(z) g$ have the same dimension. The lemma is proved. 
Remark 4.1. We note that the equation $T(z) g=g$ is usually called the analogue of the Faddeev equation for eigenfunctions of $H$.

We see that for each $z<\min \left(\sigma_{\text {two }}(H) \cup \sigma_{\text {three }}(H)\right)$, the operator $A(z)$ is positive, and there hence exists a positive square root $A^{-1 / 2}(z)$. For such $z$, we define the operator $\widehat{T}(z)=A^{-1 / 2}(z) K(z) A^{-1 / 2}(z)$, which is a symmetrized version of the Faddeev equation for eigenfunctions of $H$.

The following lemma is proved similarly to Lemma 4.2 and establishes a relation between eigenvalues of $H$ and $\widehat{T}(z)$.

Lemma 4.3. A number $z<\min \left(\sigma_{\text {two }}(H) \cup \sigma_{\text {three }}(H)\right)$ is an eigenvalue of $H$ if and only if the operator $\widehat{T}(z)$ has a unit eigenvalue and their multiplicities coincide.

\section{Proof of the main results}

In this section, we prove our main results using the statements in Secs. 3 and 4 and the Weyl criterion and the theorem on the spectrum of decomposable operators.

Proof of Theorem 2.1. Applying the theorem on the spectrum of decomposable operators and taking the equalities

$$
\sigma\left(h_{\alpha}(p)\right)=\sigma_{\text {disc }}\left(h_{\alpha}(p)\right) \cup\left[m_{\alpha}(p) ; M_{\alpha}(p)\right], \quad \bigcup_{p \in \mathbf{T}^{\nu}}\left[m_{\alpha}(p) ; M_{\alpha}(p)\right]=[m ; M]
$$

into account, we obtain the statement in Theorem 2.1.

Proof of Theorem 2.2. We first prove that $\sigma\left(H_{1}\right) \cup \sigma\left(H_{2}\right) \subset \sigma_{\text {ess }}(H)$. For this, we rewrite the set $\sigma\left(H_{1}\right) \cup \sigma\left(H_{2}\right)$ as

$$
\sigma\left(H_{1}\right) \cup \sigma\left(H_{2}\right)=\sigma_{\text {two }}\left(H_{1}\right) \cup \sigma_{\text {two }}\left(H_{2}\right) \cup \sigma_{\text {three }}\left(H_{1}\right) .
$$

The inclusion $\sigma_{\text {three }}\left(H_{1}\right) \subset \sigma_{\text {ess }}(H)$ is proved similarly to how this was done in [14].

Let $I_{1}, I_{2}$, and $\mathcal{I}$ be unit operators respectively acting in $L_{2}\left(\mathbf{T}^{\nu}\right), L_{2}\left(\left(\mathbf{T}^{\nu}\right)^{2}\right)$, and $L_{2}^{(4)}\left(\mathbf{T}^{\nu}\right)$. We first prove that $\sigma_{\text {two }}\left(H_{1}\right) \subset \sigma_{\text {ess }}(H)$. For this, it is convenient to use the Weyl criterion, i.e., it suffices to construct a sequence of orthonormalized vectors $\left\{f_{n}\right\} \subset L_{2}\left(\left(\mathbf{T}^{\nu}\right)^{2}\right)$ for which $\left\|\left(H-z_{0} I_{2}\right) f_{n}\right\| \rightarrow 0$ as $n \rightarrow \infty$. Here and hereafter, the symbol $\|\cdot\|$ means the norm in the corresponding space. Let $z_{0} \in \sigma_{\text {two }}\left(H_{1}\right)$ be an arbitrary point. By Lemma 3.1, there then exists a point $p_{0} \in \mathbf{T}^{\nu}$ such that $z_{0} \in \sigma_{\text {disc }}\left(h_{1}\left(p_{0}\right)\right)$. Therefore, there exists a nonzero function $\psi \in L_{2}\left(\mathbf{T}^{\nu}\right)$ such that

$$
\left(h_{1}\left(p_{0}\right)-z_{0} I_{1}\right) \psi=0 .
$$

We set

$$
f_{n}(p, q)=\frac{\chi_{V_{n}}(p)}{\sqrt{\mu\left(V_{n}\left(p_{0}\right)\right)}} \frac{\psi(q)}{\|\psi\|},
$$

where $\chi_{V_{n}}(\cdot)$ is the characteristic function of the set

$$
V_{n}\left(p_{0}\right)=\left\{p \in \mathbf{T}^{\nu}: \frac{1}{n+1}<\left|p-p_{0}\right|<\frac{1}{n}\right\},
$$

and $\mu\left(V_{n}\left(p_{0}\right)\right)$ is the Lebesgue measure of $V_{n}\left(p_{0}\right)$. It is easy to verify that $\left\{f_{n}\right\}$ is an orthonormalized system. We show that with $z_{0} \in \sigma_{\text {two }}\left(H_{1}\right)$,

$$
\lim _{n \rightarrow+\infty}\left\|\left(H-z_{0} I_{2}\right) f_{n}\right\|=0
$$


holds for the system $\left\{f_{n}\right\}$. We note that

$$
\begin{aligned}
\left\|\left(H-z_{0} I_{2}\right) f_{n}\right\|^{2}= & \frac{1}{\|\psi\|^{2}} \int \frac{\chi_{V_{n}}(p)}{\mu\left(V_{n}\left(p_{0}\right)\right)}\left[\int \mid\left(w(p, q)-z_{0}\right) \psi(q)-\right. \\
& \left.-\left.\int v_{1}(q, s) \psi(s) d s\right|^{2} d q\right] d p+\int\left|\int \frac{\chi_{V_{n}}(s) v_{2}(p, s)}{\sqrt{\mu\left(V_{n}\left(p_{0}\right)\right)}} d s\right|^{2} d p .
\end{aligned}
$$

Then there exists a number $C>0$ such that the second term can be estimated via $C \mu\left(V_{n}\left(p_{0}\right)\right)$, which by the construction of the set $V_{n}\left(p_{0}\right)$ tends to zero as $n \rightarrow+\infty$. We estimate the first term via

$$
\int \frac{\chi_{V_{n}}(p)}{\mu\left(V_{n}\left(p_{0}\right)\right)}\left\|h_{1}(p)-z_{0} I_{1}\right\|^{2} d p \leq \sup _{p \in V_{n}\left(p_{0}\right)}\left\|h_{1}(p)-z_{0} I_{1}\right\|^{2} .
$$

By (5.1), the estimate tends to zero as $n \rightarrow+\infty$. Hence, $z_{0} \in \sigma_{\text {ess }}(H)$. Because $z_{0} \in \sigma_{\text {two }}\left(H_{1}\right)$ is an arbitrary point, it follows that $\sigma_{\text {two }}\left(H_{1}\right) \subset \sigma_{\text {ess }}(H)$. The inclusion $\sigma_{\text {two }}\left(H_{2}\right) \subset \sigma_{\text {ess }}(H)$ is proved similarly. We have thus proved that $\sigma\left(H_{1}\right) \cup \sigma\left(H_{2}\right) \subset \sigma_{\text {ess }}(H)$.

We now prove the inverse inclusion, $\sigma_{\text {ess }}(H) \subset \sigma\left(H_{1}\right) \cup \sigma\left(H_{2}\right)$. Because the operator $K(z)$ is compact and the operator $A^{-1}(z)$ is bounded, $T(z)$ is an analytic function in $\mathbf{C} \backslash\left(\sigma\left(H_{1}\right) \cup \sigma\left(H_{2}\right)\right)$ with values in a compact set. By the self-adjointness of $H$ and Lemma 4.2, the operator-valued function $(\mathcal{I}-T(z))^{-1}$ exists for all $\operatorname{Im} z \neq 0$. From the Fredholm analytic theorem (see Theorem XIII.13 in [1]), we conclude that the operator-valued function $(\mathcal{I}-T(z))^{-1}$ exists everywhere on $\mathbf{C} \backslash\left(\sigma\left(H_{1}\right) \cup \sigma\left(H_{2}\right)\right)$ except a discrete set $S$, where it has a finite-rank residue. This implies that $\sigma(H) \backslash\left(\sigma\left(H_{1}\right) \cup \sigma\left(H_{2}\right)\right)$ consists of only isolated points, which can have accumulation points only at the boundary of the set $\sigma\left(H_{1}\right) \cup \sigma\left(H_{2}\right)$. Hence, we have $\sigma(H) \backslash\left(\sigma\left(H_{1}\right) \cup \sigma\left(H_{2}\right)\right) \subset \sigma(H) \backslash \sigma_{\text {ess }}(H)$, i.e., $\sigma_{\text {ess }}(H) \subset \sigma\left(H_{1}\right) \cup \sigma\left(H_{2}\right)$.

By Lemma 3.2, for any $p \in \mathbf{T}^{\nu}$, the operator $h_{\alpha}(p)$ has no more than one simple eigenvalue to the left of $m_{\alpha}$ and no more than one to the right of $M_{\alpha}$. Then by the theorem on the spectrum of decomposable operators, it follows from the definition of the set $\sigma_{\text {two }}\left(H_{\alpha}\right)$ that it consists of the union of no more than two intervals, which are located in both parts of the set $\sigma_{\text {three }}(H)$. Therefore, $\sigma_{\text {ess }}(H)$ consists of the union of no more than five intervals. Theorem 2.2 is completely proved.

Proof of Theorem 2.3. Let $\min _{p \in \mathbf{T}^{3}} \Delta_{\alpha}(p ; m) \geq 0$. It then follows from Lemma 3.3 that for any $p \in \mathbf{T}^{3}$, the operator $h_{\alpha}(p)$ has no eigenvalues to the left of $m$. By the definition of the set $\sigma_{\mathrm{two}}\left(H_{\alpha}\right)$,

$$
\sigma_{\text {two }}(H) \cap(-\infty, m)=\varnothing .
$$

a. Let $\min _{p \in \mathbf{T}^{3}} \Delta_{\alpha}(p ; M) \geq 0$. It can be shown similarly that

$$
\sigma_{\mathrm{two}}\left(H_{\alpha}\right) \cap(M, \infty)=\varnothing .
$$

Theorems 2.1 and 2.2 complete the proof of part a of Theorem 2.3 .

b. Let $\max _{p \in \mathbf{T}^{3}} \Delta_{\alpha}(p ; M)<0$. By Lemma 3.4, for any $p \in \mathbf{T}^{3}$, the operator $h_{\alpha}(p)$ has a unique simple eigenvalue $E_{\alpha}(p)>M$. Because the functions $v_{\alpha i}(\cdot), i=1,2$, and $w_{\alpha}(\cdot, \cdot)$ are continuous in their domains, the function $E_{\alpha}(\cdot)$ that sends an element $p \in \mathbf{T}^{3}$ to the eigenvalue $E_{\alpha}(p)$ is also continuous on the compact set $\mathbf{T}^{3}$. Hence, the set of values $\operatorname{Im} E_{\alpha}$ of $E_{\alpha}(\cdot)$ is an interval $\operatorname{Im} E_{\alpha}=\left[c_{\alpha} ; d_{\alpha}\right]$, where $c_{\alpha}>M$. It follows from the definition of $\sigma_{\text {two }}\left(H_{\alpha}\right)$ that

$$
\sigma_{\text {two }}\left(H_{\alpha}\right) \cap(M, \infty)=\left[c_{\alpha} ; d_{\alpha}\right], \quad c_{\alpha}>M .
$$

With Theorems 2.1 and 2.2, we obtain the proof of part $b$ of Theorem 2.3.

The other statements in Theorem 2.3 are proved similarly. The theorem is proved. 
Acknowledgments. The author is deeply grateful to G. M. Zhislin and the referee for the valuable and helpful remarks.

This paper was supported by the Deutsche Forschungsgemeinschaft (DFG Grant No. TR368/6-1).

\section{REFERENCES}

1. M. Reed and B. Simon, Methods of Modern Mathematical Physics, Vol. 4, Analysis of Operators, Acad. Press, New York (1978).

2. G. M. Zhislin, Tr. Mosk. Mat. Obs., 9, 81-120 (1960).

3. W. Hunziker, Helv. Phys. Acta, 39, 451-462 (1966).

4. S. N. Lakaev and M. I. Muminov, Theor. Math. Phys., 135, 849-871 (2003).

5. S. Albeverio, S. N. Lakaev, and Z. I. Muminov, Math. Nachr., 280, 699-716 (2007); arXiv:math-ph/0312050v1 (2003).

6. S. Albeverio, S. N. Lakaev, and Z. I. Muminov, Russ. J. Math. Phys., 14, 377-387 (2007).

7. S. Albeverio, S. N. Lakaev, and R. Kh. Djumanova, Rep. Math. Phys., 63, 359-380 (2009).

8. V. Heine, M. Cohen, and D. Weaire, Theory of Pseudopotentials [in Russian], Mir, Moscow (1973).

9. T. Kh. Rasulov, Theor. Math. Phys., 163, 429-437 (2010); Erratum, 696 (2010).

10. R. L. Hall, J. Phys. A, 39, 903-912 (2006); arXiv:math-ph/0512079v1 (2005).

11. K. Chadan and R. Kobayashi, J. Phys. A, 38, 1133-1145 (2005); arXiv:math-ph/0409018v1 (2004).

12. Zh. I. Abdullaev, Uzbek. Mat. Zh., No. 1, 3-10 (1996).

13. T. Kh. Rasulov, Russ. Math., 52, 50-59 (2008).

14. Yu. Kh. Éshkabilov, Theor. Math. Phys., 149, 1497-1511 (2006). 